\title{
Recent results from LEPS and status of LEPS2
}

\author{
Masaru Yosoi ${ }^{1, *}$ \\ for the LEPS/LEPS2 Collaboration \\ ${ }^{1}$ Research Center for Nuclear Physics, Osaka University, Ibaraki 567-0047, Japan
}

\begin{abstract}
Two high-energy photon beamlines, LEPS and LEPS2, have been operated at SPring-8. In both beamlines, linearly polarized photon beams up to $2.9 \mathrm{GeV}$ produced by laser-induced backward Compton scattering from 8 $\mathrm{GeV}$ electrons have been used to study quark-nuclear physics via the photoproduction of hadrons. In this article, I present some recent results from LEPS including the coherent $\phi$ photoproduction from ${ }^{4} \mathrm{He}$ and $\gamma p \rightarrow \pi^{-} \Delta^{++}$reaction, and report on the current status of the developments of the LEPS2 solenoid spectrometer.
\end{abstract}

\section{Introduction}

A photon beam in the multi-GeV energy region is a unique tool to investigate hadrons because it acts as a flavor-independent virtual $q \bar{q}$ pair beam with $J^{P C}=1^{--}$. Furthermore, photons produced by laser-induced backward Compton scattering (BCS) have some advantages. The energy distribution is rather flat so that the backgrounds from low energy photons are much less than the Bremsstrahlung photon beam. The beam polarization is high in the wide energy range. The experiments in the Laser-Electron Photon facility at SPring-8 (LEPS) have been carried out mainly using the forward charged-particle spectrometer since 2000 [1]. The typical intensity of the photon beam was $3 \times 10^{6} / \mathrm{sec}\left(2 \times 10^{5} / \mathrm{sec}\right)$ in the energy region of 1.5 $2.4 \mathrm{GeV}(1.5-2.9 \mathrm{GeV})$ [2]. These energies are suitable to study photoproductions of the $\phi$ mesons and hyperon resonances near the thresholds. In order to realize the double polarization measurements in the next stage of the LEPS experiments, we have also been developing a polarized HD target.

The second Laser-Electron Photon beamline (LEPS2) was constructed in 2011. In the LEPS2 facility, large acceptance detectors have been prepared to measure precisely both the photoproduction process and decay process simultaneously. Since 2013, the experiment using a high-resolution and large acceptance calorimeter (BGOegg) in conjunction with a high time-resolution TOF system has been performed at LEPS2 to search for the $\eta^{\prime}$-mesicnuclei, etc. [3] After the summer in 2016, we switched the main detector system for the LEPS2 experiments from BGOegg to the large acceptance solenoid spectrometer, and have started installation of parts of detectors into the solenoid magnet and developed a new data acquisition (DAQ) system. Although the detector system has not been the full setup yet, the commissioning run with beam has been started.

\footnotetext{
*e-mail: yosoi@rcnp.osaka-u.ac.jp
} 


\section{Coherent $\phi$-meson photoproduction from ${ }^{4} \mathrm{He}$}

The photoproduction of $\phi$ mesons near the threshold is one of the main subjects of the LEPS experiments. Because the meson exchange contribution is relatively small due to the OZI suppression in case of $\phi$-mesons (almost pure $s \bar{s}$ ), the $\phi$ photoproduction is useful to get information on the gluonic interactions at low energies, although at high energies a Pomeronexchange process well explains energy dependences of cross sections in various hadronic reactions. The decay angular distributions of vector mesons with the lineally polarized beam are ideal observables to know the parity of exchange particles in the $t$-channel process.

The LEPS Collaboration measured the $\gamma p \rightarrow \phi p$ reaction at forward angles for $E_{\gamma}=$ $1.5-2.4 \mathrm{GeV}[4,5]$. The energy dependence of the differential cross sections at zero degrees shows a bump structure around $E_{\gamma}=2.1 \mathrm{GeV}$, which cannot be explained by a simple $t$ channel Pomeron, $\pi^{0}$, and $\eta$ exchange model. Recently the LEPS measurements extended the maximal energy up to $2.9 \mathrm{GeV}$ [8] and confirmed the excess from the monotonic curve as shown in the top of Fig. 1. This behavior was also observed by CLAS [6, 7]. The spin density matrix element obtained by the $\mathrm{K}^{+} \mathrm{K}^{-}$decay angular distribution shows about $30 \%$ contribution of unnatural-parity exchanges such as $\pi^{0}$ and $\eta$ in the measured energy region. On the other hand, coherent photoproduction with an isoscalar $J^{P}=0^{+}$target such as ${ }^{4} \mathrm{He}$ is very useful for studying the Pomeron or other gluonic exchange processes because the $t$-channel $\pi^{0}$, and $\eta$ exchanges are prohibited.

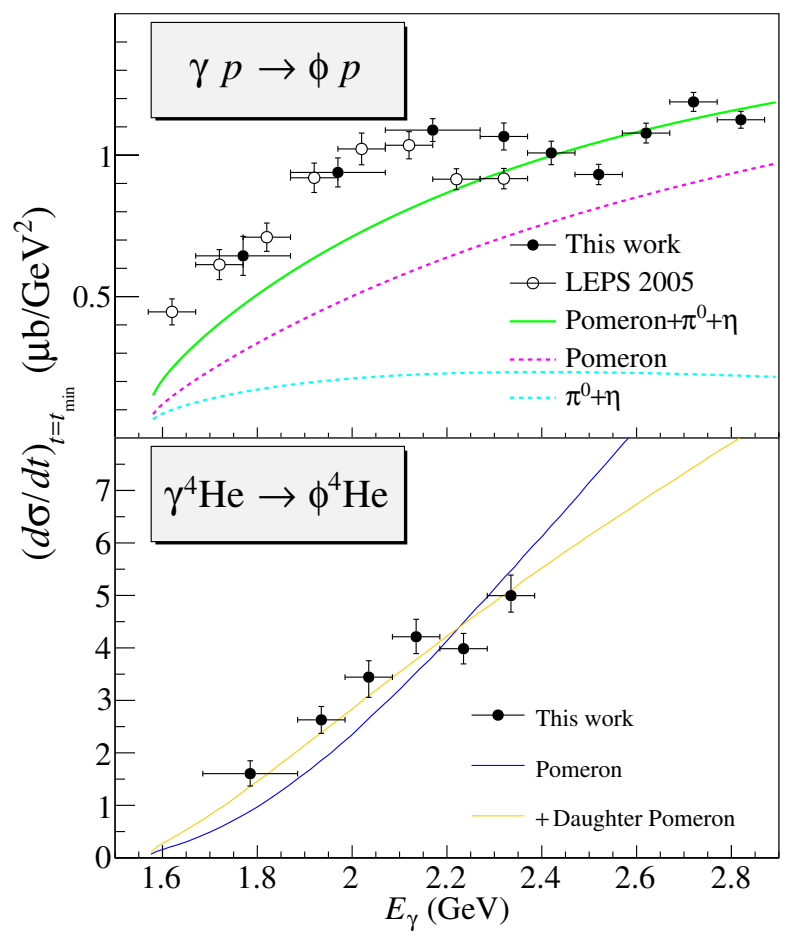

Figure 1. Energy dependences of $(d \sigma / d t)_{t=t_{\min }}$ for the $\phi$-meson photoproductions from proton (top) and ${ }^{4} \mathrm{He}$ (bottom) $[8,9]$.

We have measured the coherent $\phi$-meson photoproduction from ${ }^{4} \mathrm{He}$ near threshold for the first time [9]. Thanks to the large separation energy of the ${ }^{4} \mathrm{He}$ nucleus, coherent pro- 
duction events are cleanly separated from incoherent ones in the missing mass spectrum for the ${ }^{4} \mathrm{He}\left(\gamma, K^{+} K^{-}\right) X$ reaction after selecting the $\phi$-meson events. The obtained spin-density matrix elements demonstrate the strong dominance $(>94 \%)$ of natural parity exchanges as expected. The energy dependence of the differential cross sections at zero degrees is shown in the bottom of Fig. 1. Theoretical calculations for the coherent $\gamma^{4} \mathrm{He}$ reaction have been done according to the manner in ref. [10], in which the forward coherent cross section is described by using the natural-parity amplitudes for the elementary $\gamma \mathrm{p}$ reaction and the ${ }^{4} \mathrm{He}$ form factor. The blue curve represents the conventional Pomeron exchange model and the yellow shows the Pomeron and daughter Pomeron exchange model [11]. This suggests that some additional contribution other than the Pomeron is needed in the natural parity exchanges. Further theoretical and experimental effort will be necessary for revealing the underlying reaction mechanism.

\section{$3 \gamma p \rightarrow \pi^{-} \Delta^{++}(1232)$ reaction at forward $\pi^{-}$angles}

The photoproduction of various hadrons is important for comprehensive understanding of the production mechanisms and baryon resonances. In most of the LEPS experiments, we have used an aerogel Čerencov counter (AC) with an index of 1.03 to reject the forward $e^{+} e^{-}$ events at the trigger level to reduce the trigger rate. A part of high energy charged pions, however, also removed in this case. Since the beam intensity is low in the high energy mode using deep UV lasers, the AC can be removed or replaced by another one with a smaller index.
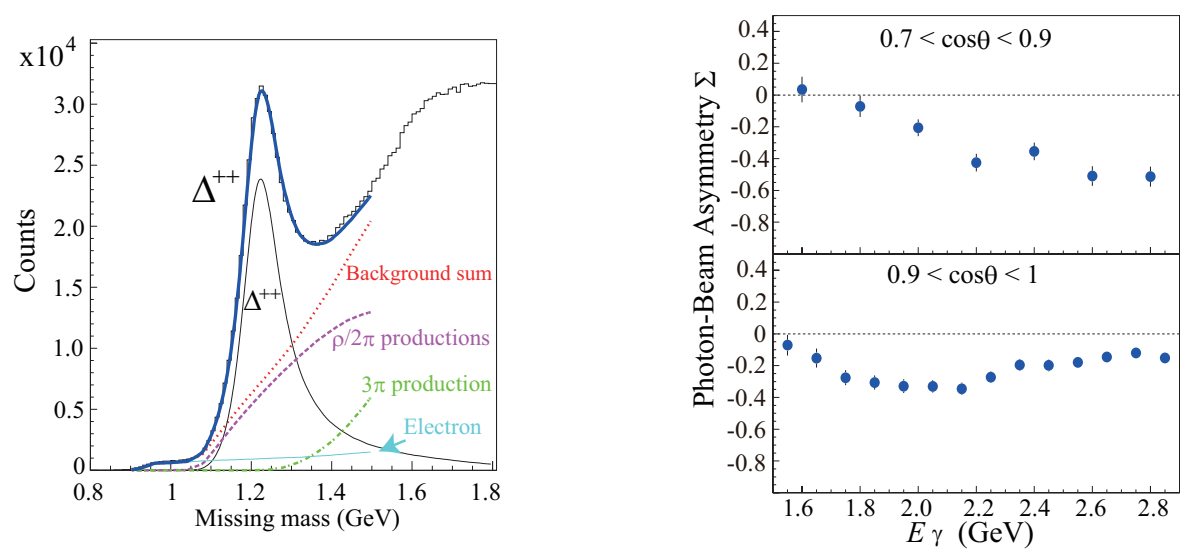

Figure 2. (Left) Missing mass of the $p\left(\gamma, \pi^{-}\right) X$ reaction for $E_{\gamma}=1.5-2.95 \mathrm{GeV}$. (Right) Photon beam asymmetries $(\Sigma)$ for the $\gamma p \rightarrow \pi^{-} \Delta^{++}$reaction in two different angle regions.

We carried out the experiment to measure high-momentum charged pions using the linearly polarized photon beam with energies of 1.5 to $2.95 \mathrm{GeV}$. The $\gamma p \rightarrow \pi^{+} n$ reaction has firstly been studied [12] and next the $\gamma p \rightarrow \pi^{-} \Delta^{++}$has been investigated. Here we shortly present the results of the first high-statistics data for the $\Delta^{++}$photoproduction. It is noting that in the latter reaction the $u \bar{u}$ quark pair is produced, while in the former the $d \bar{d}$ quark pair is produced. The missing mass of the $p\left(\gamma, \pi^{-}\right) X$ reaction is shown in the left of Fig. 2. A $\Delta^{++}$ peak is clearly observed. The $\Delta^{++}$events were selected by fitting a missing mass spectrum with the $\Delta^{++}$and background processes $(\rho / 2 \pi, 3 \pi$, and electron). In the energy dependence of the differential cross sections there is no distinct peak structure. However, the small excess 
over a theoretical prediction is observed around $E_{\gamma}=1.5-1.8 \mathrm{GeV}$, which might be due to the tail of nucleon resonances. The right of Fig. 2 shows the photon beam asymmetries of the $\pi^{-} \Delta^{++}$reaction in two different angle regions for $E_{\gamma}=1.5-2.9 \mathrm{GeV}$. The asymmetries are found negative in most of the measured kinematical region, which suggests that the unnatural parity $(\pi)$ exchange in the $t$-channel is dominant. On the other hand, the $\gamma p \rightarrow \pi^{+} n$ reaction with the $d \bar{d}$ quark pair production and the $\gamma p \rightarrow K^{+} \Lambda / \Sigma^{0}$ reactions with the $s \bar{s}$ quark pair production show the positive asymmetries $[12,14]$. The combination of these data would be helpful to understand the mechanism of hadron photoproductions comprehensively.

\section{The status of $\Theta^{+}$analysis}

The most exciting result in the LEPS experiments was the observation of a possible candidate of the narrow resonance state with a positive strangeness $(S=+1)$ [15], now called $\Theta^{+}$. The minimul quark configuration of this state is $u u d d \bar{s}$ which has no $q \bar{q}$ pair with the same flavor, while the recently discovered heavy pentaquarks $\left(P_{c}^{+}\right)$have the $c \bar{c}$ pair. Then the $\Theta^{+}$is a genuine exotic state if it exists. Although the LEPS result seemed to be supported by several experiments in other facilities, a lot of negative results were also reported with good statistics from, mostly, high energy experiments.

The LEPS Collaboration continued the $\Theta^{+}$search through the $\gamma d \rightarrow K^{+} K^{-} p n$ reaction by detecting both $K^{+}$and $K^{-}$at forward angles. Here, the $\phi$ meson contribution was rejected in the invariant mass of $K^{+} K^{-}$, and by assuming the minimum momentum spectator approximation, the quasifree reaction from neutron was selected with a good correction for the Fermi motion [16]. It is noting that the CLAS reported the null results for the same reaction, but their condition was not the quasifree process because they detected protons with more than the typical Fermi momentum. Moreover, the acceptance of the $K^{-}$detection was almost exclusive between LEPS and CLAS. We have improved the analysis to reject the quasifree process from proton by using the energy loss information in the trigger scintillation counter [17]. The new measurement with high rejection efficiency of protons ( $90 \%)$ using large scintillators has been carried out in 2013-2014. The calibrations under the condition of the blind analysis have almost been finished for the new data. Before opening the blind box, we have been studying the decomposition of the background processes carefully in order to obtain a reliable significance of the signal peak. In the MC simulation, we have included five processes, namely, $\Theta^{+}, \Lambda(1520), \phi$, and two types (scalar and vector) of non-resonant $K^{+} K^{-}$ productions. Both invariant mass distributions of $M\left(N K^{-}\right)$and $M\left(N K^{+}\right)$are simultaneously fitted, where $N$ stands for the neutron or the remaining proton not rejected. The significance of the $\Theta^{+}$peak deduced by the difference of log-likelihood with a $\Theta^{+}$peak and without it. Although it has not yet the converged value, the peak significance of around $3 \sigma$ is obtained for the previous data. The final results including the 2013-2014 data will be reported in the near future.

\section{LEPS2 solenoid spectrometer}

One of the main physics objectives in the LEPS2 project is the confirmation of the pentaquark $\Theta^{+}$with higher statistics and different detection modes from the LEPS experiment. We intended to measure the angular distribution of the differential cross sections of the $\gamma n \rightarrow K^{-} \Theta^{+}$ by detecting $\Theta^{+} \rightarrow K_{S}^{0} p \rightarrow \pi^{+} \pi^{-} p$ decay mode with a large solid angle detector at LEPS 2 . Since $\Theta^{+}$is identified in the invariant mass, this is free from the Fermi motion correction and also from the $\phi$ and no-resonant $K^{+} K^{-}$backgrounds. 
Another physics topic is the study of hyperon resonances, especially, $\Lambda(1405)$. It is well known the mass of $\Lambda(1405)$ is too light if it is an SU(3) three-quark state. Some of the theoretical models describe $\Lambda(1405)$ as a mixture of two meson-baryon states, $\pi \Sigma$ and $\bar{K} N$ [18]. The latter has higher mass and a narrow width, whose pole position is important to determine the strength of the $\bar{K} N$ interaction. This is strongly related to the existence of the $\bar{K}$-nucleus (like the $K^{-} p p$ bound state) which is one of the recent hot subjects in hadron physics. We will measure such a state by controlling the parity of exchanged particles with use of the linearly polarized beam and detecting vector $K^{*}$ meson. By selecting unnatural-parity $\left(K^{-}\right)$ exchange, we can study the resonance-shape of the $\Lambda(1405)$ in comparison with those measured by $K^{+} \Lambda(1405)$ photoproduction [19]. For this measurement, we need to detect all decay products from $K^{*}$ and $\Lambda(1405)$.
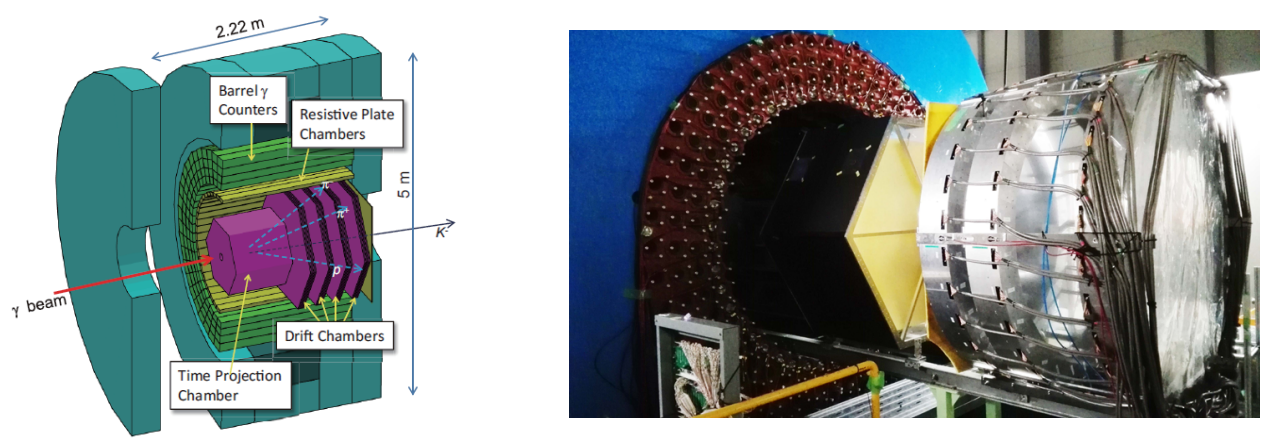

Figure 3. (Left) Schematic drawing of the LEPS2 solenoid spectrometer. (Right) Photograph of the Time Projection Chamber (TPC) and three Drift Chambers (DCs) just before being installed inside the solenoid magnet.

A schematic view of the LEPS2 solenoid spectrometer is illustrated in the left of Fig. 3. All detectors are installed in a large solenoid magnet, which had been used for the kaon raredecay experiment at BNL/E949 and was transported from BNL to SPring-8 in 2011. Its inner bore size is 2.22-m long and 2.96- $\mathrm{m}$ in diameter. The detector system of the LEPS2 solenoid spectrometer consists of drift chambers (DC), a time projection chamber (TPC), forward and side start counters (SC), resistive plate chambers (RPC), barrel electromagnetic calorimeters (Barrel $\gamma$ ), and aerogel Čerenkov counters (AC). Charged particles are momentum-analyzed by the magnetic field of $\sim 0.9 \mathrm{~T}$, and their trajectories are reconstructed by the position information of DCs and TPC. A photograph of the TPC and three DCs on the rails, just before being installed inside of the magnet, is shown in the right of Fig. 3.

The design value of the momentum resolution is $1 \%$ for $1 \mathrm{GeV}$ kaons in the range of polar angle $\theta>10^{\circ}$. The energy and direction of photons are measured with Barrel $\gamma$, which is a segmented sampling calorimeter stacking leads and plastic scintillators alternately. The velocity of each charged particle is measured by a time of flight (TOF) from the target to RPC and is used for the particle identification. In order to distinguish $K$ from $\pi$ at high momentum region $(>1 \mathrm{GeV} / \mathrm{c}$ ), ACs will be employed and placed between DCs. A small scintillator is placed on the beam axis just downstream of the solenoid magnet to reject $e^{+}$ and $e^{-}$in the trigger level. The trigger signals are produced by the forward SC or side SC surrounding the target in coincidence with the signal of photon tagging counters detecting recoiled electrons in BCS. Since SCs are placed in the strong magnetic field, MPPCs (multipixel photon counter) are used for the readout of the scintillation light. A time-resolution of about 300 ps has been obtained in the test experiment of SCs. The trigger rate of hadron 
production is estimated to be about $5 \mathrm{kcps}$. We have developed some new high-rate readout electronic modules, such as FADC boards for TPC and TDCs for DC. We have also been developing a new data acquisition (DAQ) system based on the DAQ-Middleware, which is a software framework for network-distributed DAQ system (http://daqmw.kek.jp/).

After installing SCs, three DCs, and TPC, we have performed a commissioning run since December, 2016. Although, unfortunately, there are still hard-ware problems in TPC, much progress in the development of DAQ system and analysis codes has been made during the commissioning run. We will continue the commissioning experiments for the LEPS2 solenoid spectrometer in 2018 to confirm the detector performance.

The authors gratefully acknowledge the staff of the SPring- 8 for their support. The experiments were performed at the BL33LEP and BL31LEP of SPring-8 with the approval of the JASRI as a contract beamline (Proposal Nos. BL33LEP/6001 and BL31LEP/6102). This work was supported in part by several JSPS KAKENHIs, the MEXT of Japan, the National Science Council of the Republic of China, and the National Research Foundation of Korea.

\section{References}

[1] T. Nakano et al., Nucl. Phys. A 684, 71c (2001)

[2] N. Muramatsu et al., Nucl. Instrum. Methods Phys. Res. A 737, 184 (2014)

[3] H. Shimizu and N. Muramatsu, Nuclear Physics News 27, 19 (2017)

[4] T. Mibe et al., Phys. Rev. Lett. 95, 182001 (2005)

[5] S. Y. Ryu et al., Phys. Rev. Lett. 116, 232001 (2016)

[6] H. Seraydaryan et al., Phys. Rev. C 89, 055206 (2014)

[7] B. Dey et al., Phys. Rev. C 89, 055208 (2014)

[8] K. Mizutani et al., Phys. Rev. C 96, 062201(R) (2017)

[9] T. Hiraiwa et al., Phys. Rev. C 97, 035208 (2018)

[10] A. I. Titov and B. Kämpher, Phys. Rev. C 76, 035202 (2007)

[11] T. Nakano and H. Toki, in Proceedings of the International Workshop on Exciting Physics with New Accelerator Facilities (World Scientific, Singapore, 1998) p. 48

[12] H. Kohri et al., Phys. Rev. C 97, 015205 (2018)

[13] H. Kohri et al., Phys. Rev. Lett. 120, 202004 (2018)

[14] S. H. Shiu et al., Phys. Rev. C 97, 015208 (2018)

[15] T. Nakano et al., Phys. Rev. Lett. 91, 012002 (2003)

[16] T. Nakano et al., Phys. Rev. C 79, 025210 (2009)

[17] Y. Kato, Few-Body Syst. 54, 1245 (2013) (FB20 Proceedings)

[18] D. Jido et al., Nucl. Phys. A 725, 181 (2003)

[19] M. Niiyama et al., Phys. Rev. C 79, 035202 (2008) 